\title{
Do Dressing Materials Influence the Healing Time after Enzymatic Debridement?
}

\author{
F. Sander*, A. Omankowsky*, F. Radtke*, EM Delmo Walter*, H. Haller**, L. \\ Branski $^{\star \star *}$, L.P. Kamolz, ${ }^{\star \star \star *}$ B. Hartmann* \\ *Zentrum für Schwerbrandverletzte mit Plastischer Chirurgie at Trauma Hospital Berlin, Germany , ${ }^{\star \star H L M e d C o n s u l t, ~}$ \\ Leonding, Austria, ${ }^{* * *}$ Shriners Burn Institute Galveston, Texas, USA, ${ }^{* \star * * K l i n i k}$ für Plastische, Ästhetische und \\ Rekonstruktive Chirurgie Graz, Austria
}

In a retrospective quality control study, the data of 56 burn patients treated by enzymatic debridement in the Trauma Hospital Berlin were reviewed. The results of 56 Patients, 42 male, and 14 females, within the years from 2014 to 2016, 41 having silicone or polylactide dressings are described. To determine the possible impact on wound healing of the different applications comparable subgroups were selected.

\section{Demographics:}

The average age was 47,0 years, no difference was found between male and female. Etiology of burns was flame burns in 40 patients, scalds in 10, contact in 1, electrical current in 4 and electrical arch in 1.

Average TBSA was $8,1 \%$ in the polylactic group and $22,9 \%$ in the silicone group $(p=0,0028)$. Abbreviated Burn Severity Index (ABSI) was on average 4,4 without significant differences between the groups. No significant difference could be found in the number of early ED (Enzymatic Debridement) for prevention of BICS (Burn Induced Compartment Syndrome) within the groups.
Indication for ED were burns of deep partial- thickness and even full thickness burns of limited extent according to the applicable rules of the company's manual after informed consent to ED Results (all patients):

ED was done after 2,2 +- 1,6, median 2,0 days after injury in a mean area of $7,0 \%$ BSA Hemoglobin differed from preED to post- ED on average for $1,3 \mathrm{~g} / \mathrm{l}$.

Residual necrosis after ED was found in $14 \%$ of all areas treated. Early pseudoeschar formed 18 areas out of 101 $(17,8 \%)$ in 12 of 56 patients, later pseudoeschar developed in 33 areas (32,7\%) in 15 additional patients.

Average healing time to $95 \%$ healing was 28,5 days in the polylactic group (data available in 20 of 22 patients) and 49,2 days in the silicone group (16 of 19) A regression formula showed the influence of the main components on healing time $=17,9+0,57^{*} \mathrm{ABSI}-2,21^{*}$ residual necrosis $(0 / 1)+23,7$ Pseudoeschar to be operated on $(0 / 1)-18,66^{*}$ early pseudoeschar $(0 / 1)+6,62^{*}$ type of dressing (1Supra/2Mepi) $R=0,5595(p=0,0436)$

\begin{tabular}{|c|c|c|c|c|c|c|c|}
\hline $\begin{array}{l}\text { Residual } \\
\text { necrosis }\end{array}$ & Material & $\begin{array}{c}\text { Early } \\
\text { pseudoesch } \\
\text { ar }\end{array}$ & $\begin{array}{c}\text { Late } \\
\text { pseudoesch } \\
\text { ar }\end{array}$ & Bacteria & Grafting & $\begin{array}{c}\text { Healed } \\
\varnothing \text { (d) }\end{array}$ & $\begin{array}{c}\text { Data } \\
\text { available/ } \\
\text { Data total }\end{array}$ \\
\hline \multirow{9}{*}{$\begin{array}{l}\frac{0}{0} \\
\frac{3}{3} \\
\frac{0}{0} \\
\frac{1}{0} \\
\frac{0}{0} \\
\frac{0}{0} \\
\frac{0}{0} \\
\frac{0}{0} \\
\frac{0}{3} \\
\frac{0}{7} \\
\frac{1}{7}\end{array}$} & \multirow{4}{*}{ 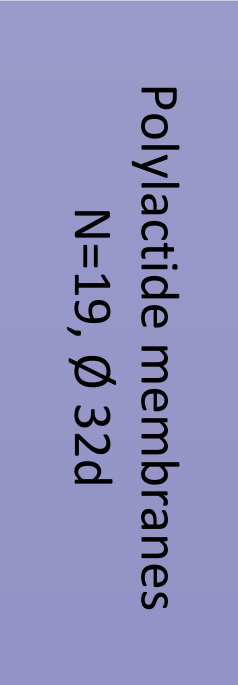 } & \multirow{3}{*}{$\begin{array}{c}\mathrm{NO} \\
\mathrm{N}=16\end{array}$} & \multirow{3}{*}{$\begin{array}{c}\text { NO } \\
n=16\end{array}$} & $\begin{array}{c}\mathrm{NO} \\
\mathrm{N}=12\end{array}$ & $\begin{array}{c}\text { NO } \\
\mathrm{n}=12\end{array}$ & 26,5 & $11 / 12$ \\
\hline & & & & \multirow{2}{*}{$\begin{array}{c}\text { YES } \\
n=4\end{array}$} & $\begin{array}{l}\mathrm{NO} \\
\mathrm{N}=1\end{array}$ & 53 & $1 / 1$ \\
\hline & & & & & $\begin{array}{l}\text { YES } \\
n=3\end{array}$ & 29 & $3 / 3$ \\
\hline & & $\begin{array}{l}\text { YES } \\
N=3\end{array}$ & $\begin{array}{l}\text { YES } \\
n=3\end{array}$ & $\begin{array}{l}\text { YES } \\
n=3\end{array}$ & $\begin{array}{l}\text { YES } \\
n=3\end{array}$ & 29 & $3 / 3$ \\
\hline & \multirow{5}{*}{ 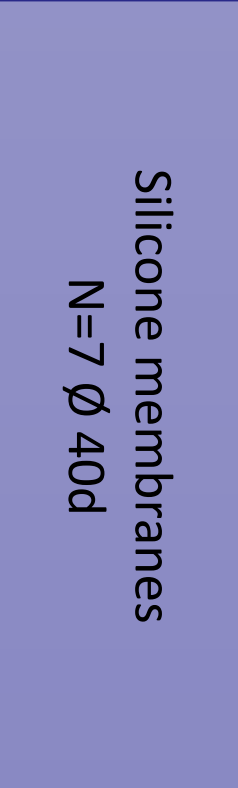 } & \multirow{4}{*}{$\begin{array}{l}\text { NO } \\
n=6\end{array}$} & $\begin{array}{l}\text { YES } \\
n=1\end{array}$ & $\begin{array}{l}\text { YES } \\
n=1\end{array}$ & $\begin{array}{l}\text { YES } \\
n=1\end{array}$ & 51 & $1 / 1$ \\
\hline & & & \multirow{3}{*}{$\begin{array}{l}\text { NO } \\
n=5\end{array}$} & \multirow{2}{*}{$\begin{array}{c}\text { YES } \\
3\end{array}$} & $\begin{array}{l}\text { YES: } \\
n=1\end{array}$ & 29 & $1 / 1$ \\
\hline & & & & & $\begin{array}{l}\text { NO } \\
n=2\end{array}$ & 22 & $1 / 2$ \\
\hline & & & & $\begin{array}{c}\mathrm{NO} \\
\mathrm{N}=2\end{array}$ & $\begin{array}{l}\text { NO } \\
n=2\end{array}$ & 48 & $1 / 2$ \\
\hline & & $\begin{array}{l}\text { YES } \\
n=1\end{array}$ & $\begin{array}{l}\text { YES } \\
n=1\end{array}$ & $\begin{array}{l}\text { YES } \\
n=1\end{array}$ & $\begin{array}{l}\text { YES } \\
n=1\end{array}$ & 51 & $1 / 1$ \\
\hline \multirow{7}{*}{$\begin{array}{l}\overline{5} \\
\hat{0} \\
\frac{3}{3} \\
\frac{0}{0} \\
\frac{0}{0} \\
0 \\
0 \\
\frac{0}{0} \\
\frac{0}{1} \\
\frac{0}{0} \\
\frac{1}{3} \\
0 \\
\frac{1}{7}\end{array}$} & \multirow{2}{*}{ 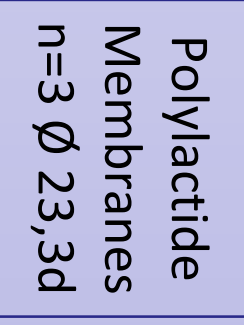 } & \multirow{2}{*}{$\begin{array}{l}\mathrm{YES} \\
\mathrm{N}=3\end{array}$} & \multirow{2}{*}{$\begin{array}{l}\text { YES } \\
n=3\end{array}$} & $\begin{array}{l}\text { YES } \\
N=2\end{array}$ & \multirow{2}{*}{$\begin{array}{l}Y E S \\
n=3\end{array}$} & \multirow{2}{*}{23,3} & \multirow{2}{*}{$3 / 3$} \\
\hline & & & & $\begin{array}{l}\text { NO } \\
n=1\end{array}$ & & & \\
\hline & \multirow{5}{*}{ 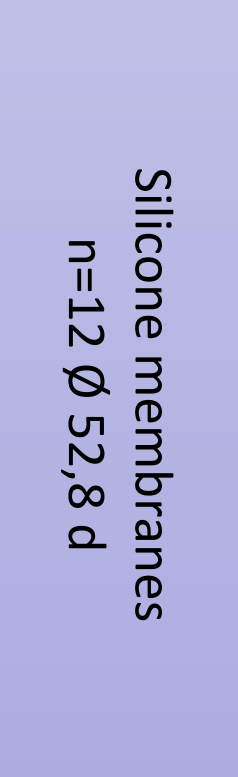 } & \multirow{3}{*}{$\begin{array}{l}\text { NO } \\
n=8\end{array}$} & \multirow{2}{*}{$\begin{array}{l}\text { YES } \\
n=7\end{array}$} & $\begin{array}{l}\text { YES } \\
n=6\end{array}$ & $\begin{array}{l}\text { YES } \\
n=6\end{array}$ & 59,2 & $6 / 6$ \\
\hline & & & & $\begin{array}{l}\text { NO } \\
n=1\end{array}$ & $\begin{array}{l}\text { NO } \\
n=1\end{array}$ & 74 & $1 / 1$ \\
\hline & & & $\begin{array}{l}\text { NO } \\
n=1\end{array}$ & $\begin{array}{l}\text { YES } \\
n=1\end{array}$ & $\begin{array}{l}\text { YES } \\
n=1\end{array}$ & 51 & $1 / 1$ \\
\hline & & \multirow{2}{*}{$\begin{array}{l}\text { YES } \\
n=4\end{array}$} & \multirow{2}{*}{$\begin{array}{l}\text { YES } \\
n=4\end{array}$} & $\begin{array}{l}Y E S \\
n=3\end{array}$ & $\begin{array}{l}\text { YES } \\
n=3\end{array}$ & 51,5 & $2 / 3$ \\
\hline & & & & $\begin{array}{l}\text { NO } \\
n=1\end{array}$ & $\begin{array}{l}\text { NO } \\
n=1\end{array}$ & 13 & $1 / 1$ \\
\hline
\end{tabular}

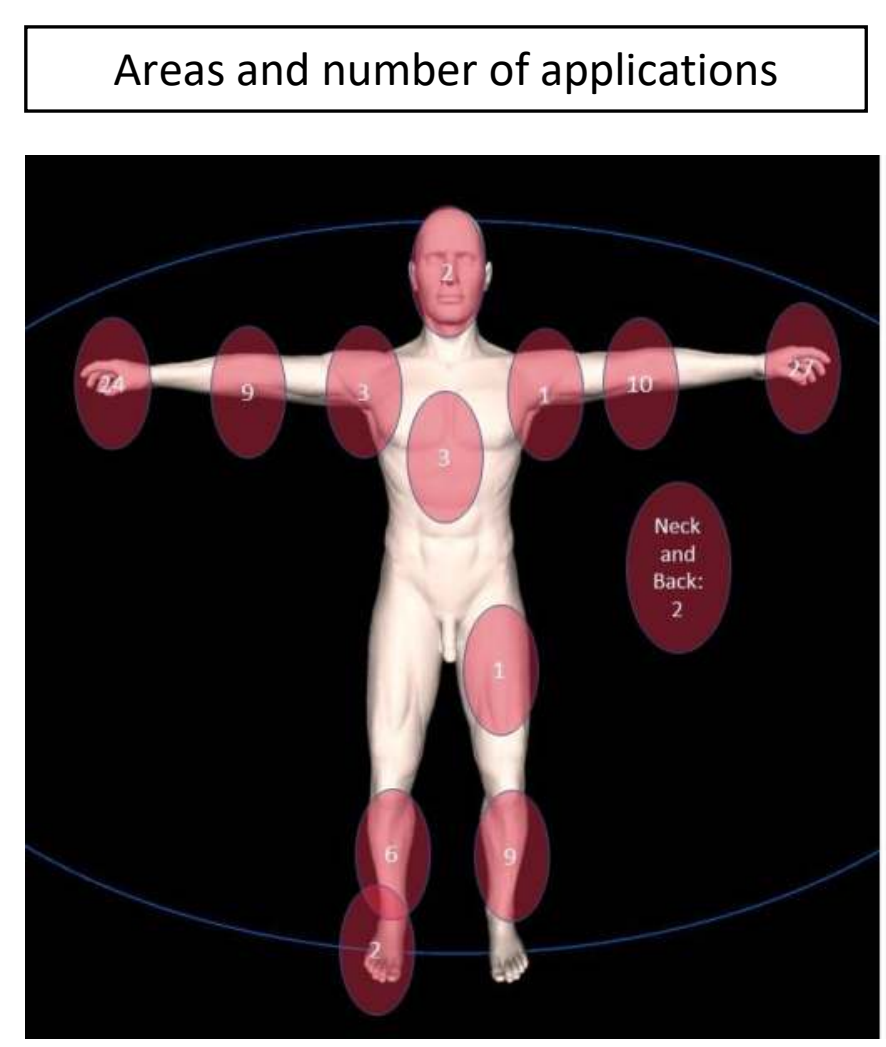

\section{Conclusion:}

As results are based on a small number of retrospective data, conclusions are limited in their validity.

Healing time was affected by severity of burn, residual necrosis and pseudoeschar, which had to be operated on, as well as by the dressing used.

Answer to the question, whether dressings do have an influence on healing time, based on these data is clearly yes.

In our study the use of polylactic membranes was associated with shorter healing time under nearly all clinical conditions.

Data also suggested a lower burn wound conversion rate in the polylactic group, which could be mediated by the biological effects of the membranes. 